\title{
Nanoscale platelet formation by monounsaturated and saturated sophorolipids under basic $\mathbf{p H}$ conditions
}

Anne-Sophie Cuvier, ${ }^{1}$ Florence Babonneau, ${ }^{1}$ Jan Berton, ${ }^{2}$ Christian V. Stevens, ${ }^{2}$ Giulia C. Fadda, ${ }^{3}$ Gérard Péhau-Arnaudet, ${ }^{4}$ Patrick Le Griel,,${ }^{1}$ Sylvain Prévost, ${ }^{5}$ Javier Perez, ${ }^{6}$ Niki Baccile ${ }^{{ }^{*}}$

[1] Sorbonne Universités, UPMC Univ Paris 06, CNRS, Collège de France UMR 7574, Chimie de la Matière Condensée de Paris, UMR 7574, F-75005 Paris, France. Corresponding author : niki.baccile@upmc.fr

[2] SynBioC, Department of Sustainable Organic Chemistry and Technology, Ghent University, Ghent, Belgium

[3] Laboratoire Léon Brillouin, LLB, CEA Saclay, F-91191 Gif-sur-Yvette Cedex, France

[4] Institut Pasteur, UMR3528, 28 Rue du Docteur Roux, F-75015, Paris, France

[5] ESRF - The European Synchrotron, High Brilliance Beamline ID02, 38043 Grenoble, France

[6] SWING, Synchrotron Soleil, BP 48, F-91192 Gif-sur-Yvette

\begin{abstract}
The self-assembly behavior of the yeast-derived bolaamphiphile sophorolipid (SL) is generally studied under acidic/neutral pH conditions, where micellar and fibrillar aggregates are commonly found according to the (un)saturation of the aliphatic chain: the cis-form, corresponding to oleic acid form of SL, spontaneously forms micelles while the saturated form, corresponding to stearic acid form of SL, rather forms chiral fibers. In this work, we explore, using small angle light and X-ray scattering (SLS, SAXS) combined with high-sensitivity cryo-Transmission Electron Microscopy (cryo-TEM), the nature of the self-assembled structures formed by these two compounds above $\mathrm{pH} 10, \mathrm{pH}$ at which they are negatively charged due to the presence of a carboxylate group. Under these conditions, they self-assemble into nanoscale platelets, despite their different molecular structure. This work shows then that the electrostatic repulsion forces generated by the $\mathrm{COO}^{-}$mainly drive the self-assembly process at basic $\mathrm{pH}$, in contrast with what it is found at $\mathrm{pH}$ below neutrality, where self-assembly is driven by van de Waals forces and hydrogen bonding, thus being in line with previous findings on carbohydrate-based gemini surfactants.
\end{abstract}




\section{Introduction}

Self-assembly properties of bipolar amphiphiles, also known as bolaamphiphiles or simply “bolas”, constitute an expanding research area since the early 1980’s. ${ }^{1,2}$ In contrast to conventional headto-tail surfactants which often self-assemble in water to form spherical, or cylindrical micelles, or bilayer structures, ${ }^{3}$ bolas are an unusual class of amphiphiles composed of two hydrophilic headgroups attached to one or two hydrophobic chains which contribute to a richer self-assembly behaviour. ${ }^{4}$ Indeed, the shape of the aggregates depends on different parameters such as the ability to form hydrogen bonds, van der Waals forces, electrostatic interactions between neighboring groups and the head-to-spacer diameter ratio of the amphiphile. Synthetic bolas based on carbohydrates, nucleotides, amino acids, and polymers have been widely reported in literature and the excellent reviews of Fuhrhop et al. and Meister et al. ${ }^{1}$ sum up the state of the art. Many bolas experience an uncommon self-organization in aqueous media because they tend to form highly interconnected objects like (nano)tubes, ${ }^{5}$ rods and tubules, ${ }^{6,7}$ fibers, ${ }^{8}$ sheets, ${ }^{9,10}$ membranes and discs or platelets. ${ }^{11}$

Among these morphologies, flat nanoscale objects like (nano)sheets and platelets exhibit large surface areas and unique properties. Rubio et al. ${ }^{12,13}$ have shown that phospholipid bicelles can incorporate amphiphilic and lypophilic anti-inflammatory drugs whose delivery and penetration through the skin could be successfully controlled. The reviews by Carmona-Ribeiro and Barbosa-Barros et al. emphazise the use of bicellar objects and discs for dermal and biomedical applications in general and illustrate the effects of lipid micelles have on the skin. ${ }^{14,15}$ These examples show the importance of studying nanoscale soft flat objects and their use in various fields such as medicine, electronics and membranes. Hereafter we will show that objects of flat morphology can be obtained from biobased, yeastderived, bolaform biosurfactants through their self-assembly in aqueous media.

Sophorolipids (SL) are a class of yeast-derived bolaform glycolipids the production of which is entirely biobased, using renewable feedstocks, like glucose and fatty acids, and yeasts (e.g., Starmerella bombicola) as bioreactor, with production yields up to $300 \mathrm{~g} / \mathrm{L}$. ${ }^{16}$ The asymmetric structure comprising a bulky sophorose headgroup and a smaller $\mathrm{pH}$ responsive carboxylic acid tail group both attached to a hydrophobic C18-alkyl chain renders this glycolipid particularly attractive with regard to self-assembly. Similar chemically-derived bolaform glycolipids were reported, for instance, to form nanotubes, ${ }^{17}$ whose mechanism of formation and applications have been recently reviewed. ${ }^{18}$ Therefore, sophorolipids are expected to lead to a broad range of morphologies and some of the reported ones are, for instance, micelles, vesicles, bilayers, but also more complex aggregates like ribbons, tubes and lamellae. ${ }^{19,20,21,22,23}$ 


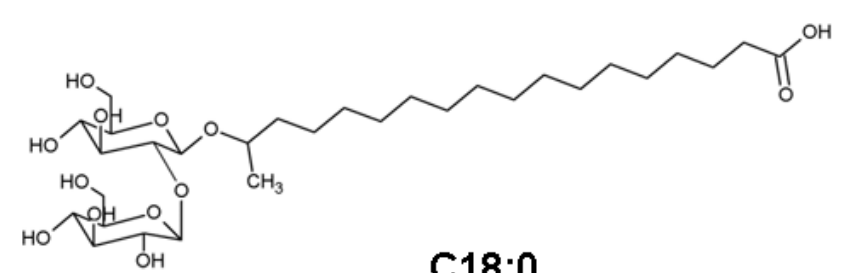

C18:0

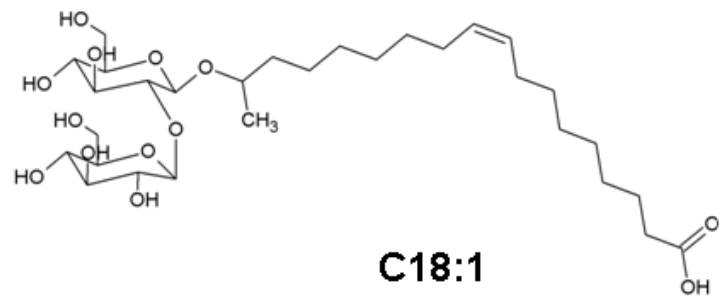

C18:1

Figure 1 - Structure of the saturated C18:0 and monounsaturated C18:1 sophorolipid molecules

These interesting aggregation properties have been subjected to a variety of studies with regard to several physico-chemical parameters, like $\mathrm{pH}$ and time ${ }^{24,25,26,27}$ and applications ranging from material science to biology have been explored; for instance, sophorolipid micelles are biodegradable templates to generate porosity in silica thin films, ${ }^{28,29}$ or to stabilize inorganic nanoparticles. ${ }^{30,31}$ Sophorolipids were also reported to have anticancer as well as antimicrobial properties, which can be used for medical applications (drug delivery, encapsulation). ${ }^{23,32,33,34,35}$ Finally, this molecule is attractive as an alternative and ecological detergent. ${ }^{36}$ The broad possibilities of applications show that this natural glycolipid has a great potential and therefore needs to be studied with regard to its self-assembly behavior. Several reports have concerned the self-assembly behavior in water of the acidic form of sophorolipids, even if some consensus must still be found. Since 2004, the monounsaturated form of sophorolipids (Figure 1) was believed to form giant ribbons at $\mathrm{pH}=2 .{ }^{24}$ Upon increasing $\mathrm{pH}$, the yield of the ribbons decreases and smaller aggregates formed in aqueous solution. In our and Penfold's groups, we have also studied this compound under similar conditions but we found the formation of nanometer-sized micelles, instead. ${ }^{25,37}$ In particular, upon increasing the $\mathrm{pH}$ above 10 , we did put in evidence the formation of large, ill-defined, structures. ${ }^{25}$ Dhasaiyan et al. have also studied the monounsaturated (C18:1) and fully saturated (C18:0) forms of sophorolipids (Figure 1) in a large $\mathrm{pH}$ range. According to them, C18:1 forms ribbon-like structures and C18:0 open sheets lying on top of each other, but no micelles were reported. In their case, the $\mathrm{pH}$ value does not seem to affect the self-assembly since differences in SEM and TEM images are negligible. ${ }^{27}$ In our group, we have recently studied the self-assembly of sophorolipid C18:0 between $\mathrm{pH} 2$ and 7.5, ${ }^{38}$ which unexpectedly self-assembles in this $\mathrm{pH}$ range to form twisted ribbons with nanoscale chirality. If compared under the same conditions, we did find that C18:1 and C18:0 have a completely different light scattering profile at $\mathrm{pH}$ below 7.5 (respectively, twisted ribbons against micelles), in spite of their very similar chemical structure (C18:0 is derived from C18:1 itself upon catalytic hydrogenation of the double bond). Interestingly, these compounds have a very similar behavior at $\mathrm{pH}$ above 7.5 , according to static light scattering. ${ }^{38}$ Incontestably, this short overview shows that there is a need to examine 
the self-assembly of sophorolipids meticulously by taking into account both the molecular structure and the $\mathrm{pH}$ and by employing the most advanced characterization techniques. As far as basic $\mathrm{pH}$ range is concerned, few studies report the self-assembly behavior of sophorolipids C18:1 and C18:0 and the data are far from being satisfactory: we ourselves have mentioned the presence of ill-defined structures, ${ }^{25}$ while Imura et al. have indicated the presence of vesicles. ${ }^{23}$

The goal of the present paper is to bring some pieces of information to the complex question of how sophorolipids C18:1 and C18:0 behave at basic $\mathrm{pH}$ conditions $(\mathrm{pH}>10)$. As a starting point, we strongly believe that sophorolipid C18:1 self-assembles into several species coexisting at the same time. The first one refers to micelles/vesicles, reported by us and Imura ${ }^{23,25}$ and the other one composed of nanoscale platelets, discussed in this study for the first time. We show that platelets can only be observed using Transmission Electron Microscopy imaging under cryogenic conditions (cryo-TEM) using a high-sensitivity CCD camera. However, the proportion between micelles, platelets and possibly a free monomeric form of the compound is still unknown. Secondly, we show here that sophorolipid C18:0 forms aggregates with very similar morphological features as the ones found for C18:1. This is quite unexpected if one compares it to the different structures (micelles versus twisted ribbons) that these compounds form at neutral/acidic $\mathrm{pH}$. In terms of the mechanisms of formation, this study indicates that for both compounds, the negative charge of the carboxylate end group drives the self-assembly properties at basic $\mathrm{pH}$ towards similar structures, thus drastically reducing the effect of both dispersion forces and hydrogen-bonding, which play a much stronger role under neutral/acidic $\mathrm{pH}$ conditions.

\section{Experimental}

\section{Materials}

The sophorolipid C18:1 sample was purchased by Soliance (France) in the typical lactone/acidic ( 80/20) mixture (Sopholiance_S, Batch $\mathrm{N}^{\circ} 11103 \mathrm{~A}$ ). A pure form of the acidic form of sophorolipid C18:1 was obtained by alkaline hydrolysis, according to method 2 described in Ref. 39, and the ${ }^{1} \mathrm{H}$ NMR spectrum of which is given in Figure S1 in the Supporting Information. This product was used to produce the acidic fully saturated form, referred to as sophorolipid C18:0. Saturation was performed by a hydrogenation reaction under hydrogen atmosphere (5 bar) using supported Pd as a solid catalyst. Details of this reaction and corresponding ${ }^{1} \mathrm{H}$ NMR spectra (disappearance of the $\mathrm{H}_{9,10}$ signal belonging to the $\mathrm{CH}=\mathrm{CH}$ bond at 5.2-5.3 ppm) have been presented in ref. 38. As a general observation on purity, one 
should nevertheless note the following. Our compounds have a very low content of residual fatty acids (less than $5 \mathrm{~mol} \%$ by ${ }^{1} \mathrm{H}$ NMR). Nevertheless, it is well-known that biobased glycolipids can contain residual congeners and in the case of sophorolipids the most common ones contain a C16 and/or diinsaturated fatty acid chains, for instance. If these are unavoidable, one can try to keep them as low as possible. According to the ${ }^{1} \mathrm{H}$ NMR spectrum presented in Figure S1, one can state that the majority of our sample is constituted by the C18:1 form of sophorolipid, however, the slight excess of the anomeric protons $\left(\mathrm{H}_{1}{ }^{\prime}, \mathrm{H}_{1}{ }^{\prime}\right.$, integral $\left.=1.92\right)$ in sophorose with respect to the $\mathrm{C}=\mathrm{C}\left(\mathrm{H}_{9,10}\right.$, integral $\left.=1.70\right)$ double bond (ratio $=1.13$ ) may indicate the spurious presence of unidentified congeners, as also detected in HPLC chromatograms presented in ref. 38 for both C18:1 and C18:0 sophorolipids. HPLC confirms that these two molecules constitute the majority of our samples ( $>90 \%)$. In this work, we make the hypothesis that the congeners contamination and residual fatty acids do not have a sensible influence on the C18:1 and C18:0 self-assembly properties.

\section{Sample preparation}

Samples are prepared by either mixing the desired amount of sophorolipids in the given volume of $\mathrm{mQ}$ water and then adding $10-15 \mu \mathrm{L}$ of $\mathrm{NaOH} 5 \mathrm{M}$ to increase the $\mathrm{pH}$ to 11 or they have been dissolved directly in a basic solution for an equilibrium $\mathrm{pH}$ of 11.6. $\mathrm{pH}$ is then reduced to 11 using $0.1 \mathrm{M}-1 \mathrm{M} \mathrm{HCl}$ solution, according to the sophorolipid concentration ( $0.5 \mathrm{w} \%$ or $5 \mathrm{w} \%)$. Both methods are equivalent as we did not observe specific changes on the sophorolipid self-assembly. Whatever the type of sophorolipid, at $\mathrm{pH} 11$ the $0.5 \mathrm{w} \%$ solution should be clear (OD600 below 0.05 ) while the $5 \mathrm{w} \%$ one should be slightly turbid (OD600 below 0.20). In both cases, aged solutions tend to be mildly turbid (OD600 above 0.20 ).

\section{Experimental techniques}

Transmission electron microscopy experiments under cryogenic conditions (cryo-TEM) were performed on two different microscopes. High-sensitivity experiments: FEI Tecnai F20 at the PFMU, Institut Pasteur (Paris, France). The microscope operates at $200 \mathrm{kV}$ and a Gatan Ultrascan US4000 CCD camera $4 \mathrm{k} x 4 \mathrm{k}, 15 \mu \mathrm{m}$ pixel size, was used to acquire the image. Lower-sensitivity experiments: FEI Tecnai 120 Twin microscope operating at $120 \mathrm{kV}$ equipped with a Gatan Orius SC1000 CCD numeric

camera $4 \mathrm{k} \times$ x 4k. The sample holder was a Gatan Cryoholder (Gatan 626DH, Gatan). On both microscopes, DigitalMicrograph ${ }^{\mathrm{TM}}$ software was used for image acquisition. Cryofixation was either done on a EMGP, Leica (Austria) instrument or on a homemade cryo-fixation device. The solutions were 
deposited on glow discharged holey carbon coated TEM copper grids (Quantifoil R2/2, Germany). Excess solution was removed and the grid was immediately plunged into liquid ethane at $-180^{\circ} \mathrm{C}$. All grids were kept at liquid nitrogen temperature throughout all experimentation.

Small Angle Neutron Scattering (SANS) was performed at the Léon Brillouin Laboratory (LLB, Orphée Reactor, Gif-sur-Yvette, France) on the PACE beamline. The spectrometer configuration was adjusted to cover three different q-ranges. High-q range: $2.90 \times 10^{-2} \AA^{-1}<\mathrm{q}<3.00 \times 10^{-1} \AA^{-1}$ a sample to detector distance, $\mathrm{D}$, of $1.0 \mathrm{~m}$, neutron wavelength, $\lambda$, of $6 \AA$. Medium-q range: $6.90 \times 10^{-3} \AA^{-1}<\mathrm{q}<$ $7.30 \times 10^{-2} \AA^{-1}, \mathrm{D}=4.7 \mathrm{~m}, \lambda=6 \AA$. Low-q range: $2.4 \cdot 10^{-3} \AA^{-1}<\mathrm{q}<2.6 \cdot 10^{-2} \AA^{-1}, \mathrm{D}=4.7 \mathrm{~m}, \lambda=17 \AA$. $\mathrm{q}$ is the wavevector and it is defined as $\mathrm{q}=\frac{4 \pi}{\lambda} \sin (\theta)$, where $2 \theta$ is the scattering angle between the incident and the scattered neutron beams. All samples were introduced in a $2 \mathrm{~mm}$ quartz cell and studied at $\mathrm{T}=25^{\circ} \mathrm{C}$. Data treatment was done with the PAsiNET.MAT software package provided at the beamline and available free of charge. The scattering intensity is obtained from the determination of the number of neutrons in the incident beam and the detector cell solid angle. Data were corrected for the ambient background, empty cell scattering, neutron beam transmission and detector efficiency and normalized to the neutron beam flux to get the scattered intensity I(q) in absolute units according to Ref. 40. Incoherent signal was substracted by measuring the background value at high-q values.

Small Angle X-ray Scattering (SAXS) has been done on the high brilliance ID02 beamline at the ESRF synchrotron in Grenoble, France and SWING beamline at Soleil synchrotron in Saint-Aubin, France. The beam energy is set to $12.6 \mathrm{KeV}$ and the high-q region is explored using a sample-to-detector distance of $1 \mathrm{~m}$ while the low-q region is explored using a sample-to-detector distance of $6 \mathrm{~m}$. Experiments have been done both in a static and dynamic regime to probe the stability of the system and reproducibility under different mixing conditions. In static experiments (at SWING beamline) the solutions at $\mathrm{pH} 11$ are prepared ex-situ (preparation of a solution at $\mathrm{pH} 11.6$ and then lowered to $\mathrm{pH} 11$ using few $\mu \mathrm{L}$ of $0.1 \mathrm{M}-1 \mathrm{M} \mathrm{HCl}$, according to the concentration of sophorolipid in solution) and analyzed in a quartz capillary using the same basic solution used to prepare the reference sample for background substraction. The capillary used to analyze both the background solution and the samples is never changed, so as to minimize imperfections during data treatment. Samples have been analyzed using an automated robot provided at the beamline and which allows a continuous flow of solution ( $50 \mu \mathrm{L} / \mathrm{min})$ in front of the beam so as to reduce possible beam damage. The acquisition time is set between $500 \mathrm{~ms}$ (for low-q) and 300 ms (high-q). Between 5 and 10 spectra are commonly acquired per each sample and averaged, thus keeping the experimental error bars very small. Given the environmental setup, the high-q 
region for all samples is analyzed within a 30 min timespan after about $4 \mathrm{~h}$ from sample preparation while the low-region is analyzed after about $7 \mathrm{~h}$. To overcome these delays between preparation and analysis, we have performed dynamic experiments (ID02 beamline, ESRF) using a flow-through polycarbonate 2 mm capillary connected to the sophorolipid-containing sample solution at $\mathrm{pH} 11.6$ through a peristaltic pump. The $\mathrm{pH}$ was controlled in-situ via a classical $\mathrm{KCl} \mathrm{pH-meter} \mathrm{directly} \mathrm{in} \mathrm{the} \mathrm{experimental} \mathrm{hutch} \mathrm{and}$ monitored live. $\mathrm{pH}$ changes have been obtained by using a $0.1 \mathrm{M} \mathrm{HCl}$ solution introduced via a motorcontrolled press-syringe. If the flow-through device prevents beam damage, we could only acquire 1 spectrum at each $\mathrm{pH}$ under this particular set-up, thus making the statistics more fluctuating than in the static experimental conditions. Error-bars on the ID02 experiments were calculated based on the estimated number of photons detected (accounting for the gain and quantum efficiency of the CCD and phosphor layer), assuming a Poisson statistics. The noise of the detector was accounted for by comparison of darks.

In all cases, data have been acquired using a CCD camera and integrated azimuthally to obtain a typical $\mathrm{I}(\mathrm{q})$ spectrum. Contribution of the solvent (water at pH 11.6) and capillary have been measured prior to the experiment and duly substracted during the data treatment. All data have been corrected for the transmission of the direct beam and scaled to be in absolute scale. Radius of gyration was estimated in the $0.03 \AA^{-1}<\mathrm{q}<0.13 \AA^{-1}$ region using the Guinier model $\mathrm{I}(\mathrm{q})=\mathrm{I}(0) \exp \left(\mathrm{q}^{2} \mathrm{R}_{\mathrm{g}}{ }_{\mathrm{g}} / 3\right)$ developed in the SASView software. ${ }^{41}$ Fitting of the SAXS curves has been done using classical model functions (core-shell cylinder, core-shell ellipsoid, bicelle) for the form factors described in the literature ${ }^{42}$ by mean of the SASView software. More pieces of information is given in the Supplementary Information.

Static Ligth Scattering (SLS) experiences were done on a Malvern/Amtec Macrotron spectrometer with a $\mathrm{He} / \mathrm{Ne}$ laser as light source operating at an incident power of $20 \mathrm{~mW}$ and a wavelength of $\lambda=633 \mathrm{~nm}$. The Rayleigh ratio, $R(\theta)$, is calculated after substraction of the water and toluene scattering signal. The expression of $\mathrm{R}_{\theta}$ is given in Eq.1 against the scattering angle $2 \theta$ ( $=20,30,40, \ldots, 110,120$, in degrees)

$$
\mathrm{R}(\theta)=\frac{\mathrm{I}(\theta)_{\text {sample }}-\mathrm{I}(\theta)_{\text {solvent }}}{\mathrm{I}(\theta)_{\text {Toluene }}} \frac{\mathrm{n}_{\text {solvent }}}{\mathrm{n}_{\text {toluene }}} \mathrm{R}_{\text {Toluene }} \quad \text { Eq.1 }
$$

where $I$ being the scattering intensity, $n$ the refractive index of the solvent, which are, for toluene

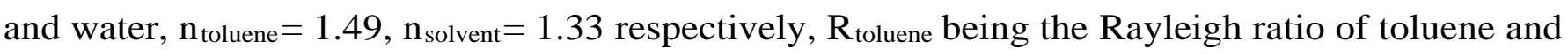
whose value is tabulated to be $0.0000138 \mathrm{~cm}^{-1}$. The expression in Eq. 1 is given in absolute scale 
and it can be plotted against the scattering vector $\mathrm{q}$ to be consistent with I(q) measured in the SANS experiments and where $\mathrm{q}\left(\AA^{-1}\right)$ is calculated as follows: $\mathrm{q}=\frac{4 \pi}{\lambda} \sin (\theta)$.

${ }^{1} \mathrm{H}$ NMR experiments were performed on a Bruker AvanceIII $300 \mathrm{MHz}$ spectrometer using a $5 \mathrm{~mm}{ }^{1} \mathrm{H}-\mathrm{X}$ BBFO probe.

\section{Results}

Small Angle X-ray Scattering experiments

Under basic $\mathrm{pH}$ conditions and at room temperature, both sophorolipids C18:1 and C18:0 given clear solutions at $0.5 \mathrm{w} \%$. The first approach to study them is to combine SAXS and SLS experiments in the $3 \times 10^{-4}<\mathrm{q}\left(\AA^{-1}\right)<1$ region, as shown in Figure 2a. For sake of clarity, the data are analyzed and compared in different q-regions, above and below the inflection point estimated at $\mathrm{q}=0.02 \AA^{-1}$. Above $\mathrm{q}=0.02 \AA^{-1}$, both compounds display a comparable SAXS signature, the interpretation of which is in first place compatible with the presence of micellar objects. Interestingly, the intensity of the plateau at $0.02<\mathrm{q}\left[\AA^{-1}\right]<0.1$ for C18:1 (empty black squares in Figure 2) is systematically lower both in SAXS (Figure 2b) and SANS (data not shown) data. Unfortunately, a straightforward interpretation of these data is difficult because of the presence of the strong overlap with the intense scattering signal at $\mathrm{q}<0.02 \AA^{-1}$ (attractive interactions). Under the hypotheses that the low-q scattering signal equally impacts the intensity at q>0.02 $\AA^{-1}$, the intensity is then proportional to the number of micelles in solution and to the electron density contrast between the micelles and the solution itself. Since C18:1 and C18:0 have been both studied at the same concentration ( $5 \mathrm{w} \%$ ) and the chemical formula of the molecules is practically the same, one expects, in the presence of micellar objects similar in shape and size, a comparable SAXS signal. If this is not the case, one can formulate the hypothesis that the conditions of study (here, basic $\mathrm{pH}$ ) are not favorable for micellar formation or that the solvent (water and $\mathrm{Na}^{+}$ions) penetrates within the micellar palisade at a different extent. In the first case, one expects a lower intensity due to the smaller number of scattering objects while in the second case, one expects a lower contrast between the solvent and the micellar objects. For core-shell micelles, as one expects here due to the chemical difference between sophorose and the aliphatic chain, the resulting signal can be even more difficult to predict due to contribution of the hydrophilic shell to the scattered signal. Since C18:1 and C18:0 have the same sophorose group, one can make a further hypothesis according to which solvent (water and $\mathrm{Na}^{+}$ions) penetration in the sophorose 
shell is similar and, for this reason, one can explain the higher SAXS intensity recorded for the C18:0 sample with a larger amount of micelles in this sample with respect to C18:1. However, a clear-cut answer cannot be provided given the large number of approximations on which this conclusion holds.

Experiments in Figure 2a have been recorded on the static sample synthesized ex-situ. To consolidate our results, we performed additional experiments using an in-situ experimental set-up, in which $\mathrm{pH}$ is changed and monitored every minute inside the same solution, which is continuously pumped through the capillary for SAXS analysis: we could then explore several $\mathrm{pH}$ values between $\mathrm{pH} 11.5$ and $\mathrm{pH} 10$ thus probing the stability of both systems in a wider $\mathrm{pH}$ range. Figure $2 \mathrm{~b}$ shows in-situ SAXS analysis in the vicinity of $\mathrm{pH} 11$. First of all, one finds that for each system, all curves have typical features of micelles in solution, their profile is very similar to the ex-situ experiments for $\mathrm{q}<0.01 \AA^{-1}$ and they are practically superimposable within the experiment error, indicating that small changes in $\mathrm{pH}$ (11.06, 10.91 and 10.74 for C18:0; 10.99, 10.89 and 10.66 for C18:1) do not really affect the nature of the objects in this regime. These conclusions are actually extendable in the entire $\mathrm{pH}$-range explored. Secondly, one finds again that the intensity of the C18:0 system is slightly higher than the C18:1, as mentioned before, but overall the curves have a very similar profile, and they are almost superimposable at $q>0.1 \AA^{-1}$. SAXS experiments allow a rough estimation of the radius of gyration, $R_{g}$, of the micellar objects. In order to minimize the impact of the structure factor due to the low-q scattering contributions to the estimation of $\mathrm{R}_{\mathrm{g}}$, we only consider the narrow $\sim 0.09 \AA^{-1}<\mathrm{q}<\sim 0.20 \AA^{-1}$ region, including the minimum of the form factor oscillation. Using these assumtpions, the estimation of $\mathrm{Rg}$ is about 17 $\AA$ for both C18:0 and C18:1, a model-independent value which is compatible with the typical size of sophorolipid cross-sectional micellar radius ( 20 $\AA$ ) found at lower $\mathrm{pH}$ values. ${ }^{26}$ However, one should probably keep in mind that this value is only an approximation of the actual $\mathrm{R}_{\mathrm{g}}$. A more detailed and critical analysis of this region of the spectrum will be provided later.

The region below $\mathrm{q}<0.02 \AA^{-1}$ is also very informative. As it was previously discussed, ${ }^{25}$ the intense scattering signal detected in C18:1 solution at basic $\mathrm{pH}$ was claimed as a direct proof for the presence of large supramolecular aggregates. To confirm these results for the C18:1, and to better investigate the low-q portion of the spectrum for the C18:0 sample, we have performed SAXS experiments at longer sample-to-detector distances $(6 \mathrm{~m})$ and complemented them with SLS experiments at $\mathrm{q}<2 \times 10^{-3} \AA^{-1}$, which nicely superimpose the SAXS data at the cut-off q-value of $2 \times 10^{-3} \AA^{-1}$, as shown in Figure 2a. Both samples provide a very strong signal increase with 
decreasing the value of the scattering angle. First of all, the Guinier plateau is not reached for the C18:0 compound and most likely not for C18:1, either. If a linear fit is operated in the entire lowq region below $\mathrm{q}<0.02 \AA^{-1}$ for both samples, one finds an average slope value of $-2.95 \pm 0.04$. However, a close look at the data indicates that a better fitting procedure can be done splitting the region into two, as shown in Figure 2a. In this case, one find two typical slope values, one in the vicinity of -2 and the other between -3 and -4 . If the strong scattering signal is consistent with the presence of large supramolecular aggregates for both compounds, the slope value close to -2 is compatible with a flat morphology ${ }^{42}$ while the value between -3 and -4 indicates the presence of surface fractal objects. ${ }^{43}$ The presence of supramolecular aggregates becomes visually evident when increasing the concentration from $0.5 \mathrm{wt} \%$ to $5 \mathrm{wt} \%$, when the solution becomes slightly turbid (images not shown).

Finally, one can compare the in-situ and ex-situ SAXS data recorded in the same q-range for a given system, as shown in Figure S2 in the Supporting Information. The comparison shows that for a given sample at $5 \mathrm{mg} / \mathrm{mL}$ and $\mathrm{pH} 11.0 \pm 0.1$, the experiment is highly reproducible in the micellar range $\left(\mathrm{q}>0.02 \AA^{-1}\right)$ : the first minimum of the oscillation is characteristic of a micellar form factor and it is slightly different between C18:0 and C18:1 samples, a hint that the electron density contrast between the micelle and the solvent is not the same for both compounds. If a detailed comparison between the form factor describing the C18:0 and C18:1 micellar systems is not in the scope of this work, one can see that the micellar structure is not influenced by the sample preparation procedure. Nevertheless, one should observe that the region at $q<0.02 \AA^{-1}$ displays slight variations in the slope according to the in-situ or ex-situ experiment, thus indicating that the formation of supramolecular aggregates is sensitive to the process kinetics. 

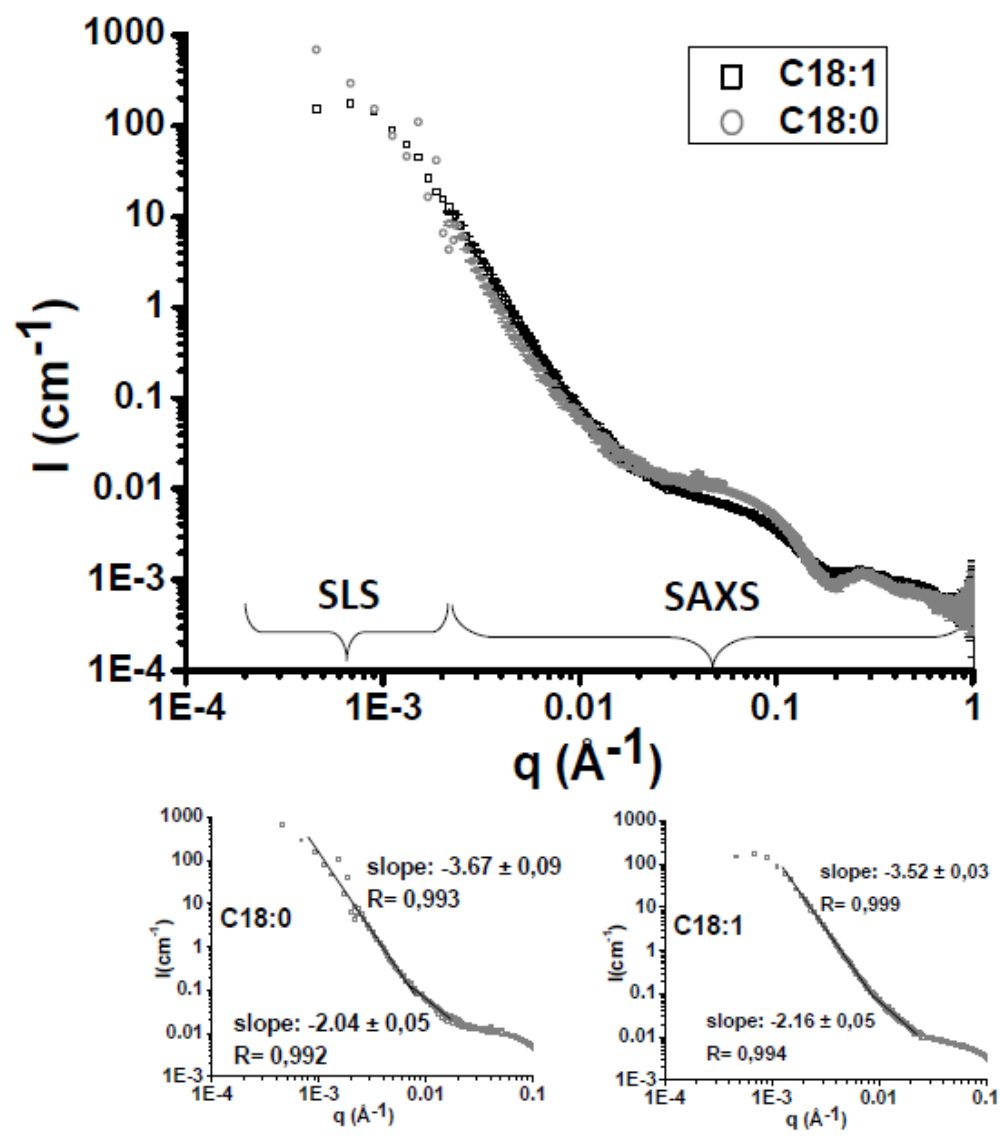

a)

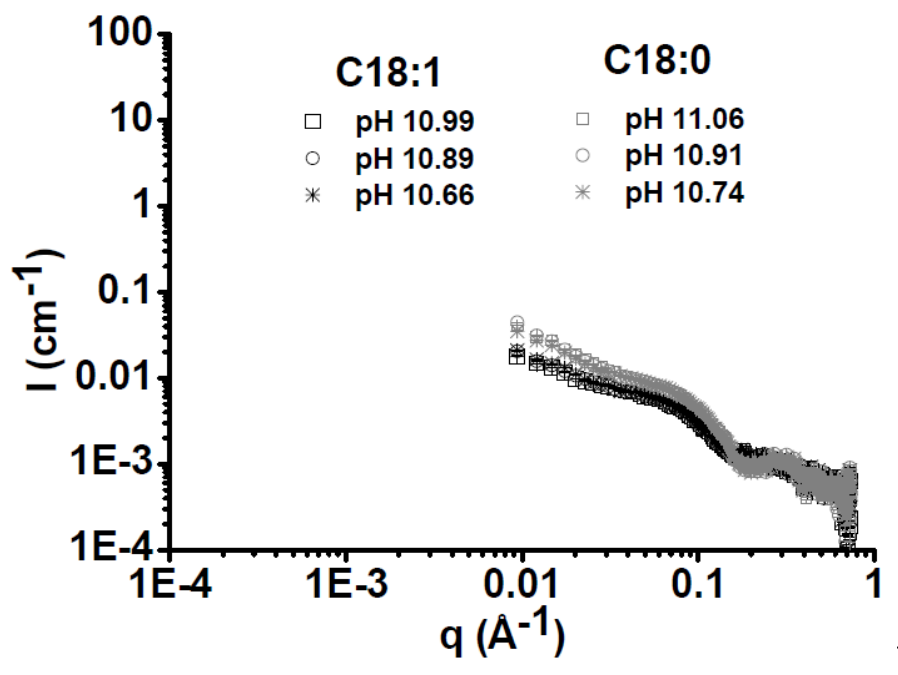

b)

Figure 2 - Log-Log representation of I(q) vs q (log-scale) of a) SAXS/SLS experiments performed on C18:0 and C18:1 sophorolipids both prepared ex-situ at $\mathrm{pH} 11$ and $5 \mathrm{mg} / \mathrm{mL}$. The highlights represent the same data extracted in the $10^{-4}<\mathrm{q}\left[\AA^{-1}\right]$ $<10^{-1}$ region and to which a linear fit has been applied. b) pH-resolved in-situ SAXS experiments performed on the same system in the vicinity of $\mathrm{pH} 11$. 


\section{Cryo-TEM experiments}

In order to study the morphology of the aggregates, cryo-TEM experiments were performed on both sophorolipid samples, C18:0 and C18:1. Although cryo-TEM has never been done on basic solutions of C18:0, preliminary images for C18:1 were already published. ${ }^{25}$ The main information that we could obtain at that time was the presence of ill-defined structures, probably composed of merged micelles/vesicles. ${ }^{26}$ However, some questions arise and the present work is meant to bring additional pieces of information to better elucidate the behavior of sophorolipids under basic $\mathrm{pH}$ conditions. First of all, we must highlight the difficulties to employ cryo-TEM for the study of both C18:1 and C18:0 samples under such $\mathrm{pH}$ conditions. This technique is known to be extremely powerful to observe soft matter in solution without altering the morphological characteristics, something which would occur for dried samples. ${ }^{44,45}$ It is particularly well-adapted for the study of surfactant systems in water, ${ }^{45}$ and it is of extreme help for heterogeneous mixtures, which constitute a clear limit for small angle scattering based experiments. However, cryo-TEM also suffers from various limitations. ${ }^{44,45}$ First of all, the thin layer of ice must be an amorphous phase, it should not contain impurities and it should be thin enough to let electrons go through, but thick enough to be stable under the electron beam for the time of observation. Various ice phases (hexagonal, cubic), or segregation phenomena can sometimes alter the observation and induce interpretation errors. ${ }^{44,45}$ The sample should be homogeneously distributed, the time of observation should be very short (seconds) and the same spot should not be observed more than once, to avoid ice melting and consequent sample evolution.

Upon studying sophorolipids in the basic $\mathrm{pH}$ region, one realizes that these conditions cannot be fulfilled all at once, thus making the use of cryo-TEM for this particular system much harder to employ, if compared to the observation of twisted ribbons, that form at lower $\mathrm{pH}$ values. ${ }^{38}$ When looking at the C18:1 sample (0.5 w\%), it is quite common to struggle to find the aggregates. We have systematically remarked that, despite a well-defined SAXS/SLS signal (Figure 2) in the lowq portion of the spectrum, it is not easy to find the clear-cut traces of the corresponding sample in cryo-TEM. The ice layer systematically appears sample-free, and in some regions of the grid one can observe the spurious presence of micelles, small vesicles ${ }^{26}$ or the entangled ill-defined structures previously reported. ${ }^{25}$ If segregation phenomena have been documented in cryo-TEM experiments, ${ }^{45}$ the mismatch between the strong SAXS/SLS signal at low-q and the lack of a homogeneous distribution on the TEM grid surface of the microscopic objects composing the sample is quite surprising. As mentioned before, the linear region below $0.02 \AA^{-1}$ in SAXS/SLS 
spectra in Figure 2a suggest the presence of both flat objects (e.g., vesicles, platelets, ribbons, disks) and surface fractals. If this may be consistent with the spurious presence of small vesicles, their finite size (below $30 \mathrm{~nm}$ in diameter) is not consistent with the typical sizes deducible from scattering experiments. These hardly reach a Guinier regime and the maximum size, $d_{\max }$, of the self-assembled objects is assumed to be equal or larger than $d_{\max }=2 \pi / q_{\min }$, where $q_{\min }$ is the minimum q-value recorded. In this case, from SLS, for $\mathrm{q}_{\min } \sim 3 \times 10^{-4} \AA^{-1}$, that is $\mathrm{d}_{\max }=21000 \AA=$ $2.1 \mu \mathrm{m}$. This order of magnitude is too large to account the presence of individual vesicles alone. For this reason, we have previously proposed the existence of ill-defined assemblies, probably constituted by conjugated/fused vesicles. Unfortunately, if the presence of such assemblies justifies the SAXS/SLS signal, it is still not a clear-cut proof for the way C18:1 may behave in solution at basic $\mathrm{pH}$ because of the unfortunate similarities they may have with typical cryo-TEM impurities, like adsorbed organic matter (frozen ethane or other), as also largely documented. ${ }^{44,45}$ Additional cryo-TEM experiments, coupled with the use of a high-sensitivity camera (Gatan US4000), have then been run, and are shown in Figure 3.

Figure 3a reports a low-magnification view of a typical cryo-TEM grid containing an amorphous layer of glassy ice filling the intra-(carbon)membrane holes. If a quick overview of the grid confirms the previous observations reported above, a more scrupulous search shows the presence of bulky aggregates captured in the ice layer, highlighted by the segmented squares in Figure 3a. If, at a first glance, these could be interpreted as impurities embedded within the ice layer, a closer look using the combination of high magnification and a high-sensitivity camera (US4000 Gatan, $15 \mu \mathrm{m}$ pixel size, $4 \mathrm{k}$ x $4 \mathrm{k}$ pixels) ${ }^{46}$ reveals a complex structure (Figure $3 \mathrm{~b}$ ) at their periphery. Whenever a cloudy aggregate as in Figure 3a is found, this is always constituted by aggregated, discrete, objects. 


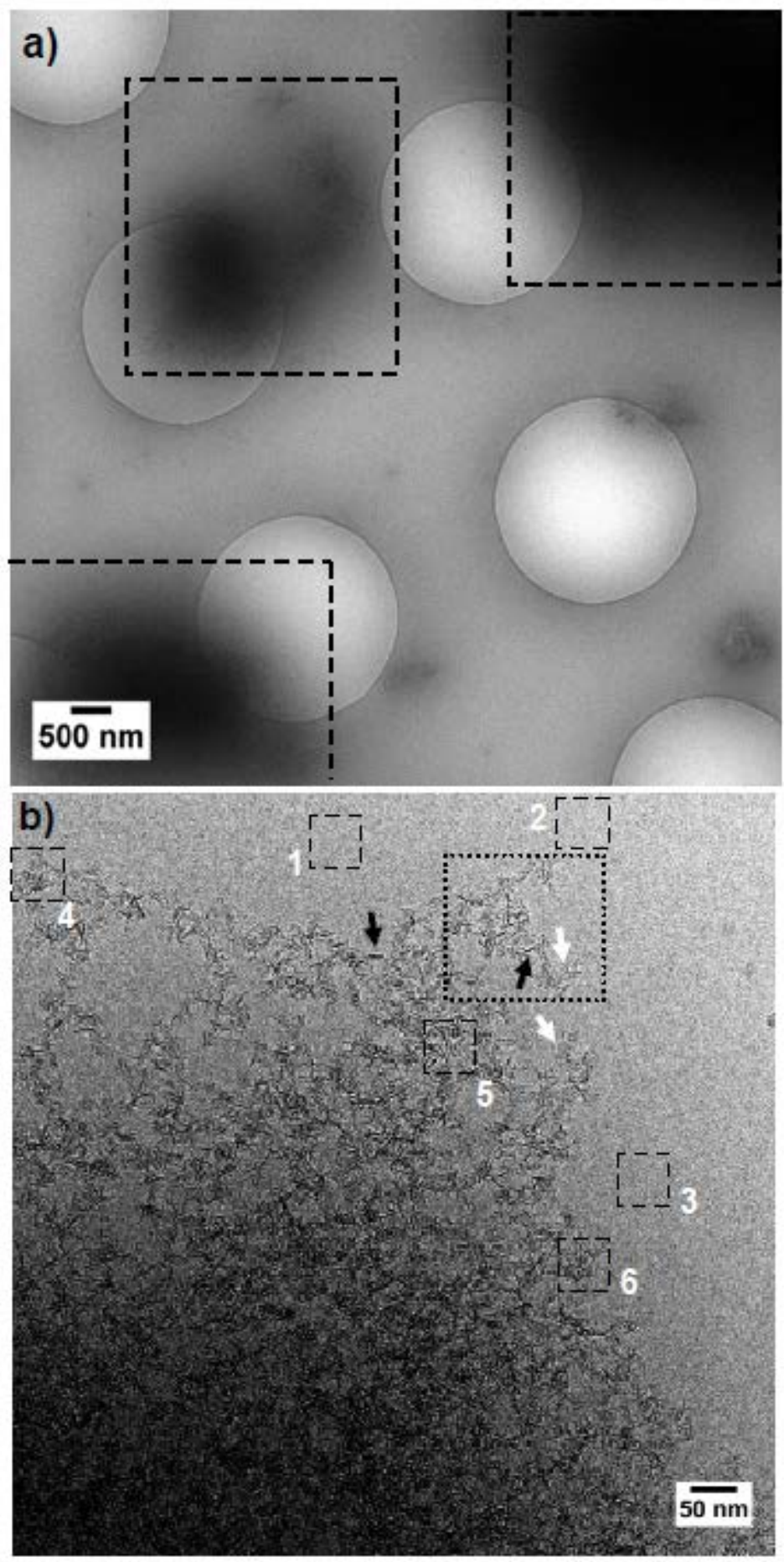



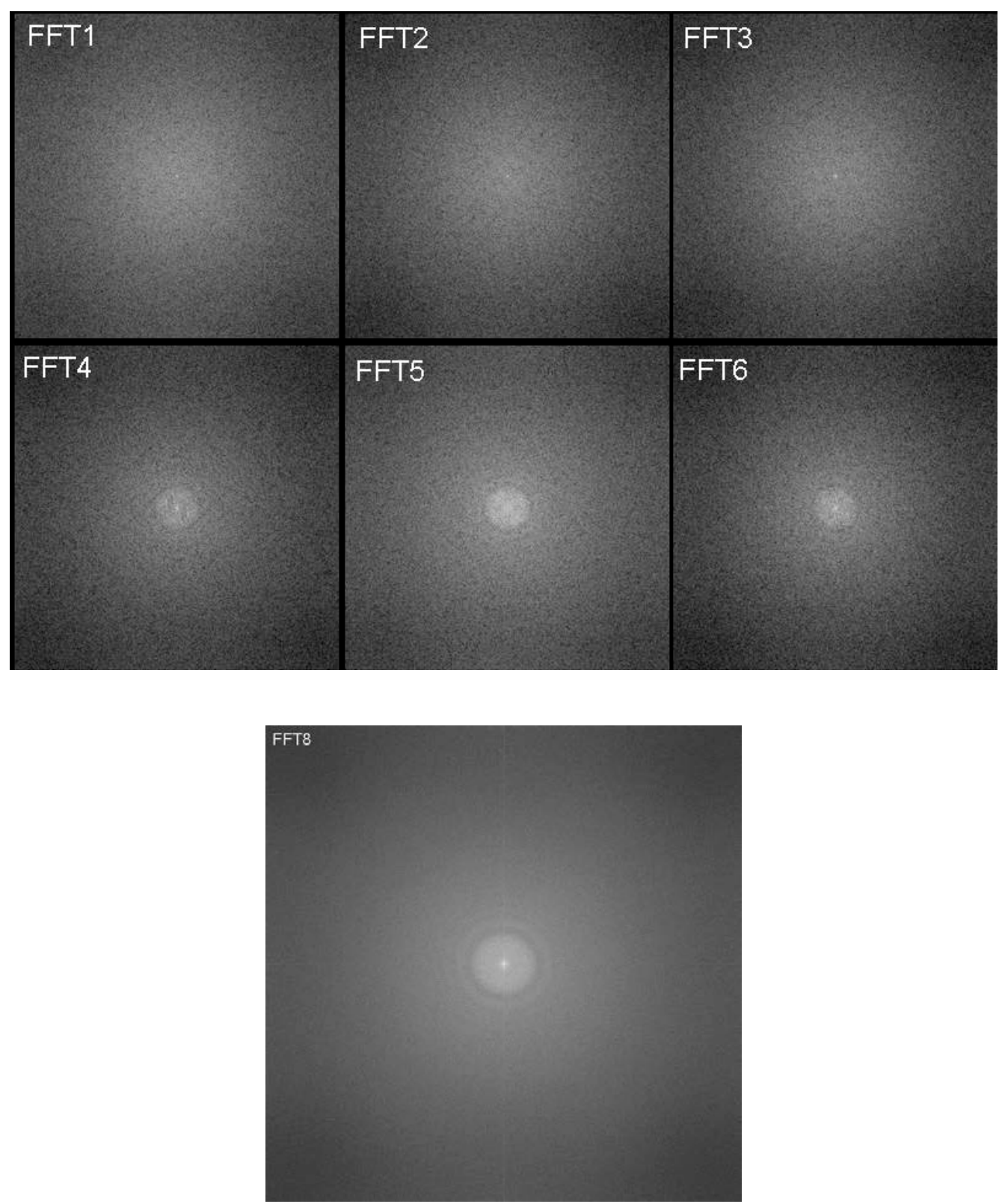

Figure 3 - a) Low-magnification cryo-TEM image showing self-assembled objects of sophorolipid C18:1 structures at pH 11 and $5 \mathrm{mg} / \mathrm{mL}$. b) High-magnification and high sensitivity (Gatan US4000) cryo-TEM image of C18:1 at pH11 and $5 \mathrm{mg} / \mathrm{mL}$. FFT1 through FFT6 images correspond to the Fast Fourier Transform (FFT) of selected numbered (1-to-6) regions given in b). FFT8 corresponds to the FFT of the entire sample region.

Figure $3 b$ shows the presence of a large number of aggregated small objects with two areas of low and high density contrast regions, as respectively indicated by white and black arrows. This peculiarity is systematically observed throughout the sample. The high-density contrast regions are constituted with objects of acicular morphology while the low-density contrast ones correspond to rather flat objects. A closer look in the large dotted square in the upper-right corner of Figure 3b is provided in Figure 4. The first question to answer is whether the sample is constituted by rods or flat objects. One simple way to answer this question is to operate a tilt of the cryo-TEM sample holder 
(here $30^{\circ}$ ). An elongated object, which always has the same cross-section, displays the same density contrast at any tilt angle. On the contrary, a flat object is poorly contrasted when observed perpendicular to its surface, while it is highly contrasted when observed on its side. The arrows in Figure 4a point to three different areas of the sample. Arrows 1 and 2 point to two, high-density, acicular regions while arrow 3 points to a flat, low-density, region. Upon a $30^{\circ}$ tilt (Figure 4a), the elongated highdensity contrast morphology pointed by arrows 1 and 2 turns into a lower density contrast one, while a higher contrast fragment appears at the location pointed by arrow 3. This seems to be a good hint that the bulky cloudy aggregate is composed of a large number of thin, flat, objects. Cryo-TEM data are in full agreement with SAXS/SLS data: first of all, the cloudy aggregates can be several micrometers in size, which is compatible with the $d_{\max }$ estimated above; secondly, the $\mathrm{I}(\mathrm{q})$ slope value measured between -3 and -4 is compatible with the cloudy aggregates, which can be considered as fractal objects from a scattering point of view; then, the additional close to -2 exponent, found by SAXS/SLS linear fit in the low-q linear region, is consistent with the flat morphology observed by cryo-TEM; however, the clear-cut determination of a specific shape is still problematic. Many literature works discuss the formation of "flat objects” from both organic ${ }^{13,47,48,49}$ and inorganic ${ }^{50,51}$ matter. They generally refer to flat sheets, platelets or bicelles, the difference between them being the size, the thickness, the number of layers, the shape, etc... Cryo-TEM is a common technique to study "flat objects" in their parent solution and it is generally very reliable when aggregates coexist with monodispersed particles. ${ }^{13,47-51}$ This is very clear, for instance, in aqueous dispersions of laponite nanoclay platelets, ${ }^{50}$ where homogeneouslydispersed individual flat objects with ill-defined shape coexist with aggregated matter. Biomimetic apatite and extracted bone also show a clear mixture of aggregated platelets, some of which are oriented in water, and individual dispersed ones. ${ }^{51}$ In both cases, the characterization of the platelets is easier if compared to our own system because of the higher electron density contrast between the mineral phase and water and the presence of isolated platelets. Lipid-based systems are also well-known to form discoidal objects and cryo-TEM is a common technique to characterize them. For instance, phospholipid-derived bicelles ${ }^{13}$ can be unambiguously described as such using cryo-TEM, if the objects are monodispersed and do not aggregate. Unfortunately, this is not the case for the sophorolipid sample at basic $\mathrm{pH}$, due to massive sample aggregation. The comparison between our images and some literature 
data shows that sophorolipids C18:1 form nanoscale platelets, ${ }^{50}$ rather than extended sheets or bicelles. The presence of local order attributed to stacking of individual platelets must be highlighted. The small numbered segmented squares in Figure 3 have been drawn to support this point. Squares 1 through 3 are voluntarily settled in the amorphous ice region while squares 4 through 6 are located in selected regions of the sample. The corresponding Fourier Transform images, presented in Figure 3 (FFT1 through FFT6), show that, as expected, no signal can be processed in squares 1 through 3, as opposed to FFT4 through FFT6. Even if the low electron density contrast between the organic matter and ice and lack of long-range order do not provide well-defined FFT images, FFT4-FFT6 are still the typical signatures of a local organization of matter; this is clear in the Fourier Transform image of the entire sample (FFT8), as it shows a typical diffraction ring which corresponds to a correlation distance in the real space of about $30( \pm 5) \AA$. This value approximately corresponds to the thickness of a single platelet, if measured directly on the cryo-TEM images. In terms of the size, the only way to estimate it is to measure the length of the acicular high-contrasted objects in regions of low particulate aggregation at the periphery of the cloudy masses. By doing so, one measures about $150( \pm 20) \AA$. 

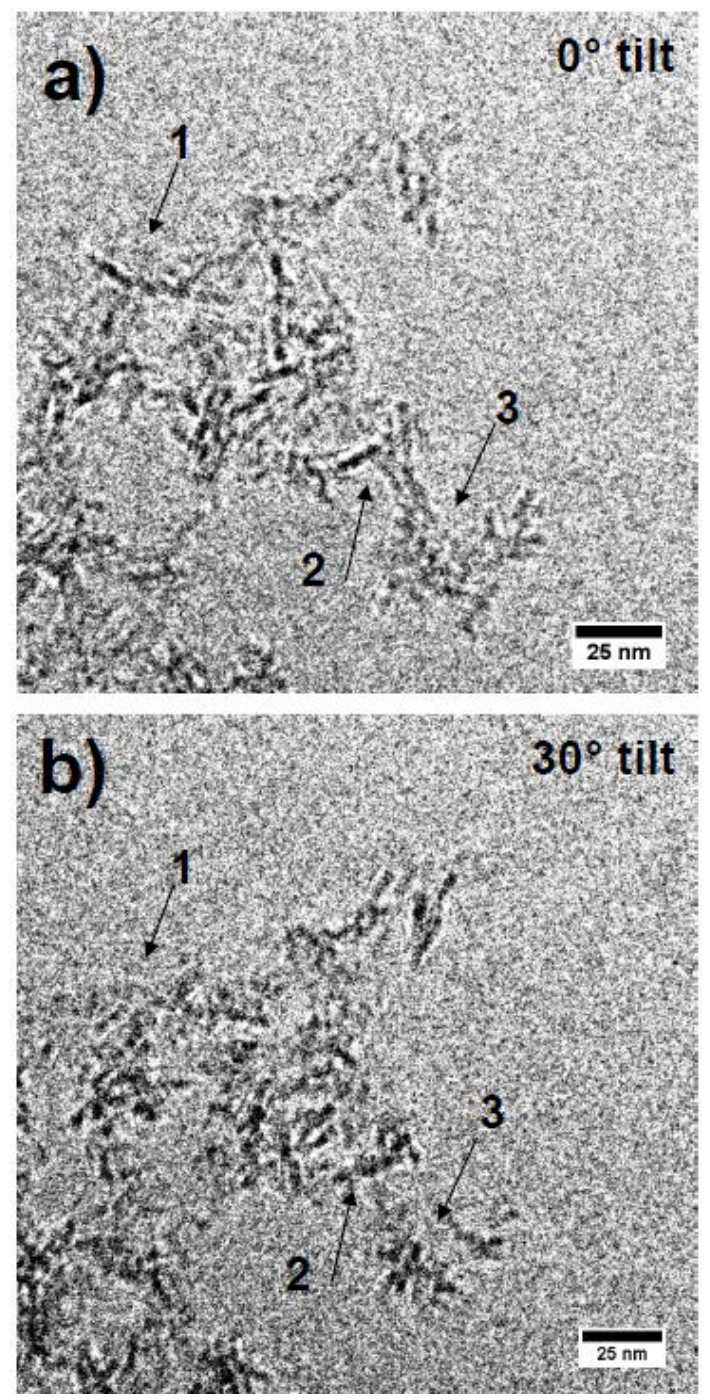

Figure 4 -High-sensitivity cryo-TEM images of C18:1 sample (pH11and $5 \mathrm{mg} / \mathrm{mL}$ ) corresponding to: a) the large dotted square in the upper-right corner of Figure 3a; b) the exact same region as a) after a tilt of the cryoTEM sample holder of $30^{\circ}$. The contrast of both images in a) and b) has been manually adjusted.

To confirm these data and to overcome the problem of the low amount of cloudy aggregates on the TEM grids, we have observed a 10-times more concentrated C18:1 sample. As expected, increasing the concentration does contribute to increase the probability of observing the aggregates. Meanwhile, concentration does not seem to alter the platelet morphology, as seen in the cryo-TEM images shown in Figure 5. Although these images are taken with a less performing SC1000 CCD camera, if compared to those presented in Figure 3 , one clearly observes the same type of multidirectional aggregation of platelets, as shown by the variation of high and low density contrasted objects in the $0^{\circ}$ and $30^{\circ}$ tilts (Figure $5 \mathrm{a}, \mathrm{b}$ and Figure 5c,d respectively) images. To support this conclusion, one can also look at the corresponding SAXS signal, presented in Figure S3a (black squares), and which shows a lowq scattering signal very similar to the one found for the $0.5 \mathrm{w} \%$ system (Figure 2). In addition to that, these scattering data show presence of a broad hump at $\mathrm{q}>0.02 \AA^{-1}$ in both SAXS and 
SANS spectra (Figure S3a and Figure S3b), indicating the presence of repulsive interactions between micelles.
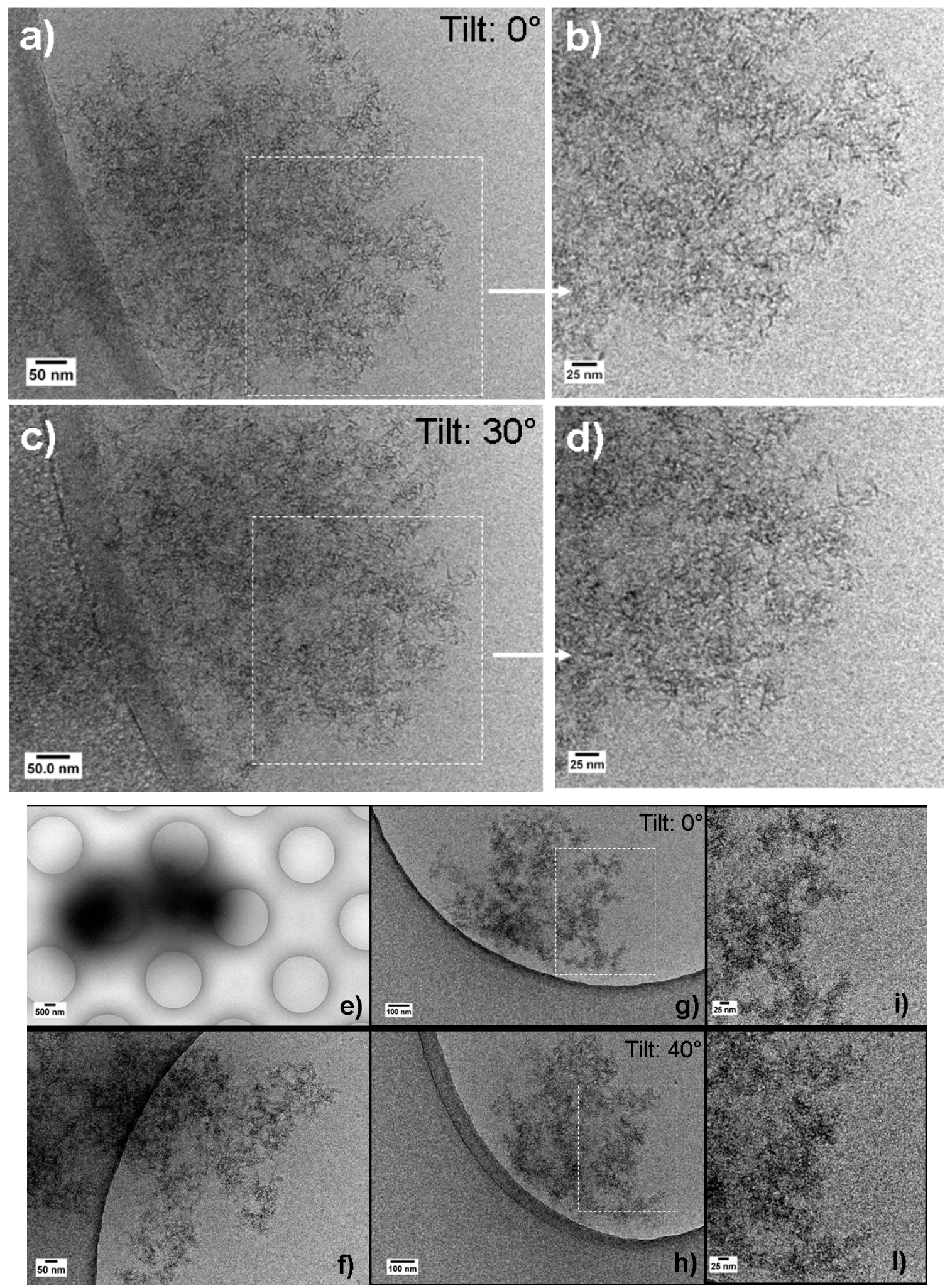

Figure 5 - a-d) Cryo-TEM image recorded at $0^{\circ}(\mathrm{a}, \mathrm{b})$ and $30^{\circ}(\mathrm{c}, \mathrm{d})$ tilt angle on a $50 \mathrm{mg} / \mathrm{mL}$ of sophorolipid C18:1 at pH11. (e-i) Cryo-TEM images recorded on a $50 \mathrm{mg} / \mathrm{mL}$ solution of sophorolipid C18:0 at pH11. Sample holder was tilted at (g,i) $0^{\circ}$ and (h,l) $40^{\circ}$. All images have been acquired on lower-sensitivity Gatan Orius SC1000 CCD camera. 
When the saturated sophorolipid C18:0 compound is analyzed (SC1000 CCD camera) at pH 11 and $0.5 \mathrm{w} \%$, one faces the same problems, as the sample can hardly be found on the TEM grid. In order to increase the probability to find the sample and to properly characterize it, we have then decided to observe a 10-times more concentrated solution, both the SAXS and SANS signals of which are also practically superimposable to the signal of C18:1 recorded at the same concentration (5 w\%), and shown in Figure S3 in the Supplementary Information. Figure 5e shows the typical cloudy aggregates which have a very close resemblance to the previously-described ones for sophorolipid C18:1. At higher magnification (Figure 5f,g), one sees that the cloud is composed of an aggregation of independent objects, the resemblance of which to a platelet morphology is also very close. Unfortunately, all our efforts to obtain better quality images were vain and even after tilting the sample holder from $0^{\circ}$ (Figure 5g,i) to $40^{\circ}$ (Figure 5h,l), it is still not possible to better describe the objects' morphology. If it is impossible, at the moment, to detail the morphology of the aggregates for the C18:0 system on the basis of our cryo-TEM experiments, the SAXS data recorded below $\mathrm{q}<0.02 \AA^{-1}$ for this compound at both $0.5 \mathrm{w} \%$ (Figure 2a) and $5 \mathrm{w} \%$ (Figure S3a) are unambiguous, and indicate the presence of the same type of flat objects observed in the C18:1 system.

\section{Discussion}

Cryo-TEM combined with SLS/SAXS show that both sophorolipids C18:0 and C18:1 self-assemble into aggregated platelets in a basic $\mathrm{pH}$ medium. This is very interesting because, as previously described, ${ }^{38}$ both sophorolipids distinguish from each other in a single $\mathrm{C}=\mathrm{C}$ bond and, in terms of their self-assembly at acidic/neutral $\mathrm{pH}$, by the formation of giant micrometer-sized ribbons with nanoscale chirality (C18:0) and nanometersized micelles (C18:1). Similar conclusions on the influence of the unsaturation on the selfassembly of sophorolipids were presented by Dhasayan et al., ${ }^{27}$ who have shown that unsaturation in the lipid moiety can drive the formation of flat sheets or vesicles in the acidic $\mathrm{pH}$ medium. However, according to the data presented here, the chemical structure of the sophorolipid does not seem to influence its self-assembly properties at basic $\mathrm{pH}$. The carboxylic acid end group is deprotonated and both compounds form very similar structures. This means that electrostatic repulsion between the negative charges overwhelms the hydrogen bonding and dispersion force effects that drive the formation of infinitely long ribbons in the case of C18:0, as found in previous works on carbohydrate-based gemini surfactants. ${ }^{52}$ In addition, the negative charge seems to drive the assembly into nanoscale 
platelets for both compounds, thus reducing the conformational influence that one unsaturated double bond may have on the final morphology. Besides the work of Imura et al., ${ }^{23}$ who argue that the sodium salt of sophorolipid C18:1 form large (200 nm in diameter) vesicles basing their assumption only on freeze-fracture arguments but no statistically-convincing small angle scattering experiments, for instance, there is no data on the behavior of sophorolipids in the basic $\mathrm{pH}$ range. For this reason, other terms of comparison must be looked at in order to understand this behavior.

Cryo-TEM images of bicelles reported in the literature ${ }^{13}$ can be compared to data obtained on our own systems. DPPC/DHPC, ${ }^{53}$ a pair of charged phospholipids, form nicely dispersed bicelles easy to observe via cryo-TEM. Another group has synthesized organic-inorganic hybrid bicelles and they can also undeniably see the flat surface by tilting the angle around the horizontal axis of the TEM grid. ${ }^{47}$ Sirieix et al. ${ }^{48}$ described the self-assembly of bolaamphiphiles with different headgroups (urocanic acid, arbutin, alcohol, carboxylic acid) and their investigations reveal that the formation of the aggregates is strongly dependent on the $\mathrm{pH}$. They report formation of vesicles and multi-layered sheets at $\mathrm{pH}>9$ and flexible fibers at lower $\mathrm{pH}$. In the presence of arbutin, a sugar-containing aromatic compound, on one side and carboxylic acid on the other, they report "geometric structures with right angles" at $\mathrm{pH}>9$, rather than small flat morphologies. Zwitterionic bolaamphiphiles with large differences in headgroup sizes (as it is also the case for sophorolipids) were studied by Graf et al. ${ }^{49}$ They have tuned the aggregation behavior of DMAPPC-C32-POH ${ }^{54}$ and DMAPPC-C32-OH, ${ }^{55}$ two charged phospholipids, by changing the symmetry of the molecule and the $\mathrm{pH}$ of the solution. While nanofibers were formed at neutral $\mathrm{pH}$, only short segmented fibers were obtained in aqueous suspensions at $\mathrm{pH} 10$ upon introduction of negative charges on DMAPPC-C32-POH. In suspensions of DMAPC-C32-OH, a bolaamphiphile where the size difference of the headgroups is comparable to our sophorolipids, a positive charge on the headgroup leads to smaller objects than in the zwitterionic state. ${ }^{49}$ Both examples clearly show that introducing charges on one headgroup strongly affects the aggregation behavior of asymmetric bolaamphiphiles, that normally tend to form smaller objects. Zwitterionic bolaphospholipids were also described by Meister et al. ${ }^{21}$, who have shown that intermolecular charge compensation leads to well defined, rigid square lamellae. In the present study, sophorolipids at basic $\mathrm{pH}$ do not express any intra-molecular charge compensation as described in ref. 21 and thus self-assembled platelets are probably less stable. According to cryo-TEM, the thickness of the platelets 
is about $30 \AA$, which is comparable to the head-to-tail conformation of a single sophorolipid molecule, which can roughly be estimated to be about $30 \AA$ using simple geometrical arguments, even if the exact values is hard to determine due to the bent configuration of oleic acid. ${ }^{25}$ For this reason, even if at the moment we cannot provide any direct spectroscopic proof, we make the hypothesis that the platelets are composed of at least a monolayer of sophorolipid molecules in a head-to-tail conformation, so to minimize repulsion between adjacent negative charges via the intercalation of a sophorose moiety. The same head-to-tail conformation is believed to be part of the twisted ribbon structure composed of sophorolipid C18:0 under neutral and acidic $\mathrm{pH}$ conditions. ${ }^{38}$

Other reports on alcohol-derivatives of alkylammonium ${ }^{56}$ and oleate $^{57}$ salt bolaamphiphiles, interesting compounds to cite because they have a similar neutralionic bolaform structure as sophorolipids do under basic $\mathrm{pH}$ conditions, have rather shown the formation of micelles, even if one would have expected flat structures from simple geometrical considerations: the $\mathrm{OH}$ group, less bulky than sophorose, would be responsible for a larger packing parameter, typical of less curbed morphologies. However, the interesting common point between our study and theirs is the fact that micelles are found to coexist with platelets, as commented in the following paragraphs. Finally, a glycolipid-based system forming platelets was also reported for the symmetrical 1-glucosamide bolaamphiphile having 11 carbon atoms as spacer between the sugar groups; ${ }^{58}$ however, in that case, hydrogen bonding was reported to be the driving force for that morphology, a fact which should less likely drive the formation of platelets in sophorolipids systems at pH 11.

Several questions are still unanswered. Why is it difficult to visualize the platelets in the cryo-TEM grids? How many types of self-assembled objects actually coexist at the same time? To provide an answer to this question one should look again at the region above $\mathrm{q}>0.02$ $\AA^{-1}$ in the SAXS data presented in Figure 2a. We did mention that this part of the spectrum characterizes the presence of micelles with a $R_{g}=17 \AA$. If platelets are not the only constituent in the sophorolipid solution, the problem concerning the frequency to observe them is probably linked to their relative proportion with respect to micelles and, probably, even non-associated monomers. However, given the cryo-TEM results, one could be tempted to attribute the scattering signal at $\mathrm{q}>0.02 \AA^{-1}$ to the contribution of the form factor characterizing the platelets themselves. At higher concentrations (5 w\%), one could even attribute the broad scattering peak (Figure S3) to possible inter-platelet interactions. To 
explore this hypothesis, we have made an attempt to use simple models to fit the SAXS pattern between $0.02<\mathrm{q}\left[\AA^{-1}\right]<0.4$ for the $0.5 \mathrm{w} \% \mathrm{C} 18: 1$ system, assuming that similar results would be obtained on the C18:0 system, given the similarity of the spectra for these two compounds. In a first approximation, we neglect the presence of repulsive interactions at lower volume fractions and we only concentrate on the impact of the form factor on the profile of the scattering curve. We have then employed the following core-shell form factors: cylinder, ellipsoid and bicelle. A critical discussion on the use of each fitting model and procedure is provided in the supporting information, while Table 1 summarizes the type of model functions used to fit the data (the SasView software package ${ }^{59}$ was used), the morphology of the objects and some key fitting parameters (all fitting values are otherwise provided with the corresponding raw data in the Supporting Information).

Table 1 - Summary of the main parameters used to fit the C18:1 0.5 w\% SAXS data above q>0.02 $\AA^{-1}$ presented in Figure 2. All values are given in $\AA$. The core-shell ellipsoid model is retained to describe the SAXS data at best. Details on the fitting procedure and parameters are given in the Supporting Information

\begin{tabular}{|c|c|c|c|c|c|c|}
\hline Model & Shape & $\chi^{2} / \mathrm{Npts}$ & $\begin{array}{c}\text { Core } \\
\text { Radius } \\
\text { (R) }\end{array}$ & $\begin{array}{l}\text { Core } \\
\text { Length } \\
\text { (L) }\end{array}$ & $\begin{array}{c}\text { Shell } \\
\text { thickness }\end{array}$ & $\begin{array}{l}\text { Rim } \\
\text { thickness } \\
\text { (shell } \\
\text { thickness at } \\
\text { edge) }\end{array}$ \\
\hline CoreShellCylinder ${ }^{60}$ & Uniform Platelet, R> L & 22399.0 & 202.6 & 30.0 & 0 & 0 \\
\hline CoreShellCylinder & Core-Shell Platelet, $\mathrm{R}>\mathrm{L}$ & 10.4 & 202.6 & 34.4 & 17.7 & 0 \\
\hline CoreShellBicelle $^{61}$ & Core-She & 9.9 & 206.6 & 32.9 & 20.2 & 5.4 \\
\hline CoreShellCylinder & Core-Shell Micelle, $\mathrm{R}<\mathrm{L}$ & 8.9 & 6.9 & 24.5 & 8.6 & - \\
\hline CoreShellEllipsoidXT $^{62}$ & $\begin{array}{l}\text { Core-Shell Ellipsoid with } \\
\text { inhomogeneous shell } \\
\text { thickness }\end{array}$ & 2.0 & 8.7 & 12.6 & $5.5^{63}$ & $15.3^{63}$ \\
\hline
\end{tabular}




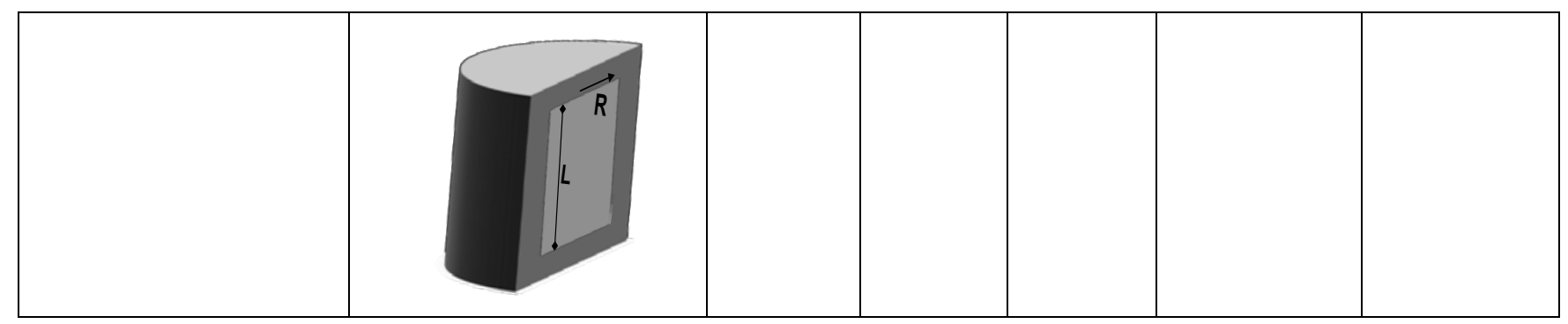

As shown in Table 1, the best results are undoubtedly obtained when using a core shell ellipsoid form factor, in agreement with the data at lower $\mathrm{pH}$ values, ${ }^{25,26,64}$ with a nonhomogenous shell size. This assumption is based on the lowest $\chi^{2} / N p t s$ value (characteristic of the good quality of the fit, see Supporting Information for more details) obtained for this model in combination with a series of criticisms when other models are used. This is detailed in the Supporting Information. The $\chi^{2} /$ Npts for the core-shell ellipsoid model is as low as 2.0 (while it can raise above 22000 for the worst case scenario using a homogenous platelet morphology) and the residuals analysis (page S13 in the SI) clearly indicates that the fitted curve nicely follows the profile of the experimental SAXS data in a wide q-range; only the shell thickness at the longitudinal edge of the micelle seems to be overestimated (15.3 $\AA$ ), even if any attempt to reduce this value always leads to non-optimized fitting functions (larger $\chi^{2} /$ Npts and non-homogeneous residuals); for instance, the best fit for a completely homogeneous shell (longitudinal and equatorial) provides a $\chi^{2} / N p t s>55$. A very small longitudinal axial shell thickness (about $1.4 \AA$ ) can also be used; however, in order to obtain an acceptable $\chi^{2} / N p t s=\sim 6$, the equatorial shell thickness must be increased to $14.4 \AA$, keeping all other parameters practically constant. In practice, good fits can only be obtained if one makes the hypothesis that the electron density distribution of the shell around the micellar core is not homogenous, as found at lower $\mathrm{pH}$ for the same compound, ${ }^{64}$ and a fact which may actually not be as surprising, if one considers the bolaform nature of sophorolipids. From the literature, it is known that phenomena like counterion condensation may strongly impact the experimental form factor in charged soft matter systems, ${ }^{65}$ as it was reported for starlike polyelectrolytes. ${ }^{66}$ At the moment, we cannot state whether or not uneven counterion condensation occurs in sophorolipid micelles, but it is not excluded that their bolaform nature may be responsible for a non-homogeneous distribution of sophorose and carboxylate groups within the micelles, as already hypothesized at lower $\mathrm{pH}^{64}$ If such a hypothesis does not seem, to the best of our knowledge, to be proposed before, older studies aiming at elucidating the structure of micelles composed of bolaform surfactants do not always agree on the intra- 
micelle molecular conformation. If recent works by Davey et al. ${ }^{56}$ and Shinde et al. ${ }^{57}$ propose, for alcohol derivatives of alkylammonium bromides and sodium oleate respectively, a homogeneous micellar structure in which the alcohol groups are in contact with the solvent, either by molecular folding or micellar crossing, older studies seem to state that alcohol- and ketone functions in alkylammonium boaamphiphile derivatives reside in the aliphatic core of the micelle, instead. ${ }^{67,68} \mathrm{We}$ then believe that, at the actual state of the art, there is large room for improvement in the precise description of bolaamphiphile micelles, but this is not the goal of this work.

Despite the above, the main drawback of the core-shell ellipsoid analysis is that the low-q scattering $\left(\mathrm{q}<0.02 \AA^{-1}\right)$ attributed to the platelets is obviously not taken into account: one must then consider that the SAXS spectrum comes from the contribution of several signals, ellipsoidal micelles and aggregated platelets. Unfortunately, quantification of the relative proportion of platelets against micelles is a harsh task but we believe that time plays an important role, the freshly-prepared system being characterized by a larger share of micelles, which are possibly consumed in favor of platelet formation. Even if a timedependent study is out of the scope of this work, one can observe a sensible difference in the slopes of SAXS experiments between the in-situ (Figure 2b, no aging) and ex-situ (Figure 2a, 3h/4h aging) curves at $\mathrm{q}<0.02 \AA^{-1}$ for both C18:1 and C18:0 experiments, as also directly highlighted in the in-situ vs. ex-situ data comparison in Figure S2 in the Supporting Information; such a difference could suggest a time-dependent evolution of the platelet aggregates, as proposed before ${ }^{26}$ for the C18:1 system. Bare-eye observation is also of help, because freshly-prepared solutions, as studied here, generally appear clear at $0.5 \mathrm{w} \%$ and slightly turbid at 5 w\%; at longer aging times (several hours or days), solutions become turbid.

As far as the platelet nanoscale size (approximately 30 x $150 \AA$ ) is concerned, it may not be surprising to observe such small objects. If the hypothesis of the head-to-tail conformation is valid, which easily justifies the $30 \AA$ thickness, the limited surface extension should be correlated to the possibility of platelet growth in the planar direction. Many experimental and theoretical studies have been developed to explain the elasticity and instability of lipid membranes. Geometrical molecular packing parameters, ${ }^{69,70}$ electrostatic instabilities, ${ }^{71,72}$ charge distributions at the membrane surface ${ }^{73} \mathrm{pH}$-dependent molecular packing ${ }^{74}$ are some of the mechanisms that have been reported and that explain the growth, or growth-limiting (via membrane bending), of lipid membranes. Even if most of these studies have been developed for charged phospholipids-based systems, the basic principles should 
apply to the sophorolipid case. Here, the subtle combination of electrostatic repulsion among $\mathrm{COO}^{-}$groups and bulkiness of the sophorose head are able to destabilize the platelet surface and could be responsible for a local increase in the lipid membrane curvature. These effects have been modeled to predict vesicle formation in anionic/cationic lipid mixtures. ${ }^{70}$ The alternate head-to-tail arrangement is responsible for the spacing between two adjacent $\mathrm{COO}^{-}$ groups, thus limiting electrostatic repulsions between them and promoting membrane growth. However, at the same time, the bulky sophorose headgroup could be responsible for headhead repulsion due steric hindrance and increase the local curvature, a well-known phenomenon. ${ }^{75} \mathrm{~A}$ way to test this hypothesis will probably be the use of sophorolipidderivatives containing one single glucose unit, so to decrease the effective headgroup volume. In this case, one expects the formation of larger membranes or vesicles. These experiments are currently undergoing. The possibility to form sophorose-water and sophorose-sophorose hydrogen bonding networks may also play a key role to destabilize the membrane and promote formation of nanoscale platelets. However, as predicted by Yuet, ${ }^{70}$ and as found experimentally in many real systems, ${ }^{73,78,76}$ physico-chemical parameters like temperature, concentration, time, ionic strength, nature of the counterions may strongly affect the free energy of the system and, eventually, the final morphology. Finally, it is possible that the cloudy aggregates are actually composed of a continuous surface of interconnected nanoscale platelets. This is supported both by cryo-TEM images and the surface fractal SAXS signal. Inter-platelet interaction can occur either via membrane interpenetration through typical diffusion limited or reaction limited colloid aggregation mechanisms ${ }^{77}$ or via watermediation. The first one has been documented, among other, in the time and temperature aggregation phenomena of lipid nanodiscs into unilamellar vesicles; ${ }^{76,78}$ the second one is reported, for instance, in the aggregation of micron-sized biomimetic hydroxyapatite platelets. ${ }^{51}$ Whatever the mechanism, sophorose-sophorose or sophorose-water hydrogen bonding could induce aggregation. Unfortunately, we have no way at the moment to further clarify this point. Finally, Figure 6 summarizes the self-assembly similarities and differences between the C18:1 and C18:0 sophorolipids. 


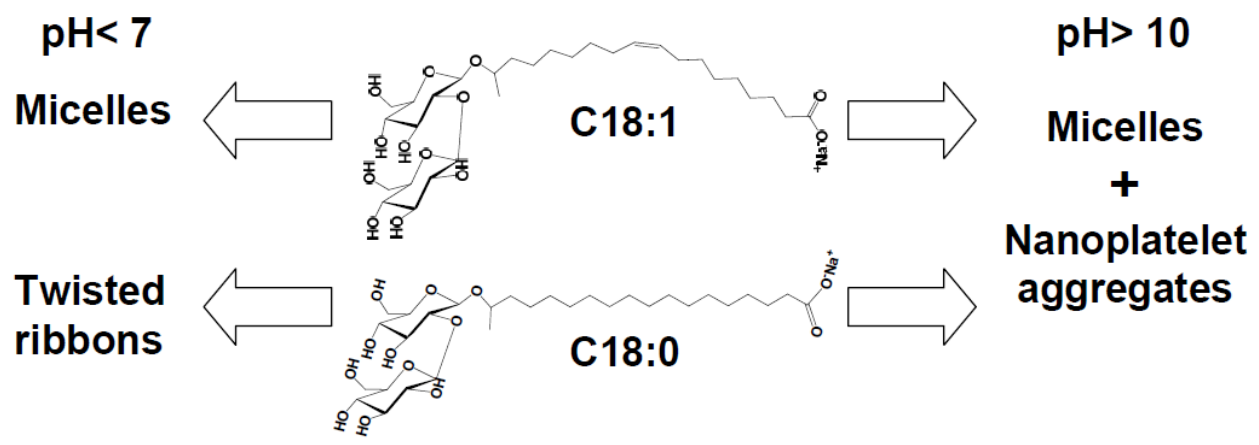

Figure 6 - Scheme summarizing the self-assembly behavior of C18:1 and C18:0 sophorolipids with pH. At basic $\mathrm{pH}$, the presence of the $\mathrm{COO}^{-}$group drives the formation of micelles and nanoscale platelet aggregates for both compounds. Upon decreasing the $\mathrm{pH}$ value, the $\mathrm{COOH}$ groups are responsible for the formation of twisted ribbons for the $\mathrm{C18:0}$ and micelles for the $\mathrm{C18:1}$ sophorolipids.

As a last note, one can remark that cryo-TEM experiments in Figure 3 show an inter-platelet order, as pointed out by the Fourier transform of the images. If the measured inter-platelet distance lies between 30 and $40 \AA$, no specific trace of a diffraction peak directly associated to the inter-platelet interaction can be observed in the SAXS experiments. We believe that the reason for this depends both on the relatively low amount of platelet aggregates in solution and on the fact that the expected q-range at which the diffraction peak would be expected is $0.16<\mathrm{q}\left[\AA^{-1}\right]<$ 0.20 , that is at the minimum of the form factor oscillation. In other words, by an unfortunate coincidence, the diffraction peak could be hidden by the ellipsoid form factor, a classical phenomenon which is known to affect the relative intensities among Bragg peaks in ordered micellar systems. ${ }^{79}$

\section{Conclusion}

In the present paper we have investigated the self-assembly of two different sophorolipid molecules under basic pH conditions. The monounsaturated (C18:1) and the saturated (C18:0) forms of sophorolipids are known to form, respectively, micelles and ribbons with nanoscale chirality at $\mathrm{pH}$ below neutrality: the presence of a double bond has a strong influence on the nature of the self-assembled species. Upon $\mathrm{pH}$ increase to 11, however, both compounds have a very similar SAXS and SLS response with a characteristics peak of micellar aggregates but also a signal which could be attributed to the presence of flat objects. Highly-performing cryo-Transmission Electron Microscopy (high-sensitivity CCD camera being used) puts in evidence the presence of aggregated platelets the size of which is about 30 x $150 \AA$. A closer look at 
the SAXS signal shows the presence of micelles which can only be described with a core-shell ellipsoid model and an uneven electron density distribution in the hydrophilic shell.

\section{Acknowledgements}

The research leading to these results has received funding from the European Community’s Seventh Framework Program (FP7/2007-2013) under Grant Agreement $\mathrm{n}^{\circ}$ Biosurfing/289219. Authors thank Dr. Jérôme Fresnais (Laboratoire PHENIX, UMR 8234m Université Pierre et Marie Curie, Paris, France) for helping on Light Scattering experiments. Dr. Francisco Fernandes (Laboratoire de Chimie de la Matière Condensée de Paris, Université Pierre et Marie Curie) is kindly acknowledged for his help on the micelle model drawings.

\section{References}

\footnotetext{
${ }^{1}$ A. Meister, A. Blume, Curr. Opin. Colloid Interf. Sci., 2007, 12, 138-147

2 J.-H. Fuhrhop, T. Wang, Chem. Rev., 2004, 104, 2901-2937

${ }^{3}$ J. N. Israelachivili, D. J. Mitchell, W. Ninham, Assem. Hydrocarb. Amphiphiles, 1975, pp. 1525-1568

${ }^{4}$ R. Nagarajan, Chem. Eng. Comm., 1986, 251-273

${ }^{5}$ a) M. Masuda, T. Shimizu, Langmuir, 2004, 20, 5969-77; b) T. Shimizu, M. Masuda, H. Minamikawa, Chem. Rev. 2005, 105, 1401-43.

${ }^{6}$ J. Sirieix, N. L. Viguerie, M. Riviere, A. Lattes, Langmuir, 2000, 16, 9221-9224

${ }^{7}$ M. Gubitosi, L. Travaglini, A. D’Annibale, N. V Pavel, J. Vázquez Tato, M. Obiols-Rabasa, S. Sennato, U. Olsson, K. Schillén, L. Galantini, Langmuir, 2014, 30, 6358-66

${ }^{8}$ R. Iwaura, K. Yoshida, M. Masuda, K. Yase, T. Shimizu, Chem. Mater, 2002, 14, 3047-3053

${ }^{9}$ K. Liu, Y. Yao, C. Wang, Y. Liu, Z. Li, X. Zhang, Chem. Eur. J., 2012, 18, 8622-8

${ }^{10}$ M. Kogiso, Y. Okada, T. Hanada, K. Yase, T. Shimizu, Biochim. Biophys.a Acta 2000, 1475, 346-352

${ }^{11}$ E. Johansson, A. Lundquist, S. Zuo, Biochim. Biophyszica Acta Biomembr., 2007, 1768, 1518-1525

${ }^{12}$ L. Rubio, G. Rodríguez, C. Alonso, M. Cócera, A. De Maza, J. L. Parra, O. López, Colloids Surf. B, Biointerf., 2012, 92, 322-326

${ }^{13}$ L. Rubio, C. Alonso, G. Rodríguez, L. Barbosa-Barros, L. Coderch, a De la Maza, J. L. Parra, O. López, Int. J. Pharm., 2010, 386, 108-13

${ }^{14}$ A. Carmona-Ribeiro, Curr. Med. Chem. 2006, 13, 1359-1370

${ }^{15}$ L. Barbosa-Barros, G. Rodríguez, C. Barba, M. Cócera, L. Rubio, J. Estelrich, C. López-Iglesias, A. de la Maza, O. López, Small, 2012, 8, 807-18

${ }^{16}$ U. Rau, S. Hammen, R. Heckmann, V. Wray, S. Lang, Ind. Crop. Prod., 2001, 13, 85-92

${ }^{17}$ N. Kameta, H. Minamikawa, M. Masuda, Soft Matter, 2011, 7, 4539-4561

${ }^{18}$ T. G. Barclay, K. Constantopoulos, J. Matisons, Chem. Rev., 2014, 114, 10217-10291

19 T. Wang, J. Jiang, Y. Liu, Z. Li, M. Liu, Langmuir, 2010, 26, 18694-700

20 T. Shimizu, M. Masuda, J. Am. Chem. Soc., 1997, 119, 2812-2818

${ }^{21}$ A. Meister, A. Blume, Curr. Opin. Colloid Interf. Sci., 2007, 12, 138-147

22 J. Schneider, C. Messerschmidt, A. Schulz, M. Gnade, B. Schade, P. Luger, P. Bombicz, V. Hubert, J.-H. Fuhrhop, Langmuir, 2000, 16, 8575-8584

${ }^{23}$ T. Imura, T. Morita, T. Fukuoka, M. Ryu, K. Igarashi, Y. Hirata, D. Kitamoto, J. Oleo Sci., 2014, 63, 141-147

${ }^{24}$ S. Zhou, C. Xu, J. Wang, W. Gao, R. Akhverdiyeva, V. Shah, R. Gross, Langmuir, 2004, 20, 7926-32

${ }^{25}$ N. Baccile, F. Babonneau, J. Jestin, G. Pehau-Arnaudet, I. Van Bogaert, ACS Nano, 2012, 6, 4763-4776

${ }^{26}$ N. Baccile, J. S. Pedersen, G. Pehau-Arnaudet, I. N. A. Van Bogaert, Soft Matter, 2013, 9, 4911

${ }^{27}$ P. Dhasaiyan, A. Banerjee, N. Visaveliya, B. L. V Prasad, Chem. Asian J., 2013, 8, 369-72
} 
${ }^{28}$ N. Baccile, N. Nassif, L. Malfatti, I. N. A. Van Bogaert, W. Soetaert, G. Pehau-Arnaudet, F. Babonneau, Green Chem., 2010, 12, 1564

${ }^{29}$ N. Baccile, T. Fontecave, C. Boissière, I. N. A. Van Bogaert, J. Phys. Chem. C, 2013, 117, 23899-23907

${ }^{30}$ M. Kasture, S. Singh, P. Patel, P. A. Joy, A. A. Prabhune, C. V. Ramana, B. L. V. Prasad, Langmuir, 2007, 23, 11409

${ }^{31}$ N. Baccile, R. Noiville, L. Stievano, I. N. A. Van Bogaert, Phys. Chem. Chem. Phys., 2013, 15, 1606

${ }^{32}$ I. N. A. Van Bogaert, J. Zhang, W. Soetaert, Process Biochem., 2011, 46, 821-833

${ }^{33}$ L. Zhang, P. Somasundaran, S. K. Singh, A. P. Felse, R. Gross, Colloids Surf. A Physicochem. Eng. Asp., 2004, 240, 75-82

${ }^{34}$ J. N. Sleiman, S. A. Kohlhoff, P. M. Roblin, S. Wallner, R. Gross, M. R. Hammerschlag, M. E. Zenilman, M. H. Bluth, Ann Clin Lab Sci, 2009, 39, 60

${ }^{35}$ I. M. Banat, A. Franzetti, I. Gandolfi, G. Bestetti, M. G. Martinotti, L. Fracchia, T. J. Smyth, R. Marchant, Appl. Microbiol. Biotech., 2010, 87, 427

${ }^{36}$ R. Marchant, I. M. Banat, Biotechnol. Lett., 2012, 34, 1597-605

${ }^{37}$ J. Penfold, M. Chen, R. K. Thomas, C. Dong, T. J. P. Smyth, A. Perfumo, R. Marchant, I. M. Banat, P. Stevenson, A. Parry, I. Tucker, I. Grillo, Langmuir, 2011, 27, 8867-8877

${ }^{38}$ A.-S. Cuvier, J. Berton, C. V. Stevens, G. C. Fadda, F. Babonneau, I. N. A. Van Bogaert, W. Soetaert, G. Pehau-Arnaudet, N. Baccile, Soft Matter, 2014, 10, 3950-3959

${ }^{39}$ N. Baccile, A.-S. Cuvier, C. Valotteau, I. N. A. Van Bogaert, Europ. J. Lipid Sci. Technol., 2013, 115, 1404

${ }^{40}$ A. Brulet, D. Lairez, A. Lapp, J.-P. Cotton, J. Appl. Cryst., 2007, 40, 165-177

${ }^{41}$ http://www.sasview.org

42 O. Glatter, O. Kratky, Small Angle X-ray Scattering, Academic Press: London, 1982

${ }^{43}$ J. Teixeira, J. Appl. Cryst., 1988, 21, 781-785

${ }^{44}$ D. Cheng, D.R.G. Mitchell, D-B. Shieh, F. Braet, Curr. Microsc. Contrib. Adv. Sci. Technol., 2012, Vol. 2, Formatex Research Center, A. Mendez-Vilas, Ed.

${ }^{45}$ Y. Talmon, Cryogenic Temperature Transmission Electron Microscopy in the study of surfactant systems, chapter 5 in Surfactant Sicnece Series, Vol. 83, Modern Characterization Methods of Surfactant Systems, B. P. Brinks, Ed., 1999, Marcel Dekker, Inc.

${ }^{46}$ Please refer to the camera developer website http://www.gatan.com/products/tem-imagingspectroscopy/ultrascan-camera

${ }^{47}$ K. Yasuhara, S. Miki, H. Nakazono, A. Ohta, J. Kikuchi, Chem. Commun., 2011, 47, 4691-3

48 J. Sirieix, N. L. De Viguerie, M. Rivière, A. Lattes, New. J. Chem., 2000, 24, 1043-1048

${ }^{49}$ G. Graf, S. Drescher, A. Meister, V. M. Garamus, B. Dobner, A. Blume, Soft Matter, 2013, 9, 9562

${ }^{50}$ N. Negrete Herrera, J.-M. Letoffe, J.-L. Putaux, L. David, E. Bourgeat-Lami, Langmuir, 2004, 20, 1564-1571

${ }^{51}$ Y. Wang, S. Von Euw, F. M. Fernandes, S. Cassaignon, M. Selmane, G. Laurent, G. Pehau-Arnaudet, C. Coelho, L. Bonhomme-Coury, M.-M. Giraud-Guille, F. Babonneau1, T. Azaïs, N. Nassif, Nature Mater., 2013, 12,1144-1153

52 J. E. Klijn, M. C.A. Stuart, M. Scarzello, A. Wagenaar, J. B. F. N. Engberts pH-Dependent Phase Behavior of Carbohydrate-Based Gemini Surfactants. The Effects of Carbohydrate Stereochemistry, Head Group

Hydrophilicity, and Nature of the Spacer, J. Phys. Chem. B, 2007, 111, 5204-5211

${ }^{53}$ Ddipalmitoyl phosphatidylcholine and dihexanoyl phosphatidylcholine

54 (32-\{[hydroxyl(2-hydroxyethoxy)phosphinyl]oxy\}dotriacontane-1-yl-\{2-[N-(3-dimethylaminopropyl)-N,Ndimethylammonio]ethylphosphate)

55 (32-hydroxydotriacontane-1-yl-\{2-[N-(3-dimethylaminopropyl)-N,N-dimethylammonio]ethylphosphate \})

56 T. W. Davey, W. A. Ducker, A. R. Hayman, Langmuir, 2000, 16, 2430-2435

${ }^{57}$ N. Shinde, K. S. Narayan, J. Phys. Chem., 1992, 96, 5160-5165

58 T. Shimizu, M. Masuda, J. Am. Chem. Soc., 1997, 119, 2812-2818

59 The full analytical list of the model functions is given at the developer's website http://www.sasview.org/sasview/user/models/model_functions.html

${ }^{60}$ http://www.sasview.org/sasview/user/models/model_functions.html\#coreshellcylindermodel

${ }^{61}$ http://www.sasview.org/sasview/user/models/model_functions.html\#coreshellbicellemodel

62 http://www.sasview.org/sasview/user/models/model_functions.html\#coreshellellipsoidxtmodel

${ }^{63}$ In this model, the shell thickness at equatorial and longitudinal positions is not homogeneous. By "shell thickness" we then intend it to be taken at equatorial position of the micelle, while the by term «Rim», generally employed for bicelles model, we intend the shell thickness in the longitudinal dimension of the micelle.

${ }^{64}$ S. Manet, A.-S. Cuvier, C. Valotteau, G. C. Fadda, J. Perez, E. Karakas, S. Abel, N. Baccile, J. Phys. Chem. B, 2015, Accepted

${ }^{65}$ M. Ballauff, A. Jusufi, Colloid Polym. Sci., 2006, 284, 1303-1311 
${ }^{66}$ A. Jusufi, J. Chem. Phys., 2006, 124, 044908

${ }^{67}$ F. M. Menger, J. M. Jerkunica, J. C. Johnston, J. Am. Chem. Soc., 1978, 100, 4676-4678

68 J. M. Brown, J. D. Schofield, J. Chem. Soc., Chem. Comm., 1975, 434

${ }^{69}$ W. Li, T. H. Haines, Biochemistry, 1986, 25, 7477-7483

${ }^{70}$ P. K. Yuet, D. Blankschtein, Langmuir, 1996, 12, 3802-3818

${ }^{71}$ M. Winterhalter, W. Helfrich, J. Phys. Chem., 1988, 92, 6865-6867

${ }^{72}$ M. D. Betterton, M. P. Brenner, Phys. Rev. Lett., 1999, 82, 1598-1602

${ }^{73}$ M. M. A. E. Claessens, B. F. van Oort, F. A. M. Leermakers, F. A. Hoekstra, M. A. Cohen Stuart Biophys. J., 2004, 87, 3882-3893

${ }^{74}$ H. Hauser, Proc. Nati. Acad. Sci. USA, 1989, 86, 5351-5355

75 J. Israelachvili, Intermolecular and Surface Forces, 2nd ed. Academic Press Inc., New York, 1985

${ }^{76}$ M.-P. Nieh, P. Dolinar, N. Kucerka, S. R. Kline, L. M. Debeer-Schmitt, K. C. Littrell, J. Katsaras, Langmuir, 2011, 27, 14308-14316

77 M. Y. Lint, H. M. Lindsay, D. A. Weitz, R. Klein, R. C. Ball, P. Meakin, J. Phys.: Condens. Matter, 1990, 2, 3093-3113

${ }^{78}$ S. Mahabir, D. Small, M. Li, W. Wan, N. Kučerka, K. Littrell, J. Katsaras, M.-P. Nieh, Biochim. Biophys. Acta, 2013, 1828, 1025-1035

${ }^{79}$ F. Michaux, N. Baccile, M. Impéror-Clerc, L. Malfatti, N. Folliet, C. Gervais, S. Manet, F. Meneau, J. S. Pedersen, F. Babonneau, Langmuir, 2012, 28, 17477-17493 